\title{
EXISTENCE OF NONATOMIC CHARGES
}

\author{
K. P. S. BHASKARA RAO and M. BHASKARA RAO
}

(Received 14 December 1971; revised 8 January 1977)

Communicated by P. D. Finch

\begin{abstract}
A complete characterization of Boolean algebras which admit nonatomic charges (i.e. finitely additive measures) is obtained. This also gives rise to a characterization of superatomic Boolean algebras. We also consider the problem of denseness of the set of all nonatomic charges in the space of all charges on a given Boolean algebra, equipped with a suitable topology.

Subject classification (Amer. Math. Soc. (MOS) 1970): primary 28 A 60
\end{abstract}

\section{Introduction}

There are no satisfactory necessary and sufficient conditions for a Boolean sigmaalgebra to have a nonatomic measure defined on it. However, in certain topological measure spaces such characterizations have been obtained (see Knowles (1967), Theorem 1, p. 64, and Luther (1970), Corollary 3.5, p. 458). The central problem tackled in this paper is to give characterizations of Boolean algebras admitting nonatomic charges. In Section 2 we establish the notation and give some relevant definitions. In Section 3 we give necessary and sufficient conditions for a Boolean algebra to admit a nonatomic charge (Theorem 1). One interesting characterization is that a Boolean algebra $B$ admits a nonatomic charge if and only if $B$ contains a tree (for the definition of a tree see Section 2). Another condition is given in terms of the Stone space of the Boolean algebra. These results give rise to a characterization of superatomic Boolean algebras (Corollary 2). In Section 4 we give necessary and sufficient conditions for the collection of all nonatomic probability charges to be dense in the collection of all probability charges with respect to an appropriate topology (Theorem 2). The closure of all nonatomic probability charges in general is also determined (Theorem 3). The main results obtained in this paper are based on the results of Knowles (1967) and the Stone representation theorem for Boolean algebras.

Copyright. Apart from any fair dealing for scholarly purposes as permitted under the Copyright Act, no part of this JOURNAL may be reproduced by any process without written permission from the Treasurer of the Australian Mathematical Society. 


\section{Definitions and notation}

For topological notions we generally follow Kuratowski (1966) and for notions about Boolean algebras we follow Halmos (1963). More specifically, Boolean algebras will be denoted by Roman capitals $A, B, C, \ldots$, and elements of the Boolean algebras by $a, b, c, \ldots$. Fields and sigma-fields of subsets of a set are denoted by script letters $\mathscr{F}, \mathscr{G}, \ldots$, and elements of these collections by $F, G, \ldots$.

A charge is a nonzero, real valued, nonnegative and finitely additive function defined on a Boolean algebra vanishing at the zero element of the Boolean algebra (see Taylor, 1958, p. 401). A measure is a countably additive charge whose domain of definition is either a field or a sigma-field of subsets of a set. A nonzero element $a$ of a Boolean algebra $A$ is called an atom for $A$ if $b \in A$ and $b \leqslant a$ then $b=a$ or $b=0$. If $\mu$ is a charge on $A$, an element $a \in A$ is called a $\mu$-atom if $\mu(a) \neq 0$, and if $b \leqslant a$ then $\mu(b)=\mu(a)$ or $\mu(b)=0$. A charge $\mu$ is said to be nonatomic on $A$ if $A$ contains no $\mu$-atoms.

Two Boolean algebras $A$ and $B$ are said to be isomorphic if there exists a one-to-one map from $A$ onto $B$ which preserves the operations of join and complementation.

A collection of nonzero elements $\left\{a_{i_{1}, i_{2}, \ldots i_{k}}: k \geqslant 1\right.$ and $i_{1}, i_{2}, \ldots, i_{k}$ is any finite sequence of 0 's and 1's $\}$ in a Boolean algebra $A$ is called a tree in $A$ if

(i) $a_{0} \vee a_{1}=1$,

(ii) $a_{i_{1}, i_{2}, \ldots, i_{k-1}, 0} \vee a_{i_{1}, i_{2}, \ldots, i_{k-1}, 1}=a_{i_{1}, i_{2}, \ldots, i_{k-1}}$, and

(iii) $a_{i_{1}, i_{2}, \ldots, i_{k-1}, 0} \wedge a_{i_{1}, i_{2}, \ldots k-1,1}=0$.

\section{Main results}

The following lemma is used in Theorems 1 and 2.

Lemma. Let $\mu$ be a measure on a sigma-field $\mathscr{G}$ of subsets of a set $X$ and let $\mathscr{F}$ be a field on $X$ which generates $\mathscr{G}$. If $\mu$ is nonatomic on $\mathscr{G}$, then $\mu$ is nonatomic on $\mathscr{F}$.

Proof. This result follows from the Caratheodory extension theorem.

REMARK. The converse of this lemma is not true. The following is an example. Let $X=[0,1]$. Let $\mathscr{F}$ be the field generated by $\{(a, b] \subset[3 / 8,5 / 8]: 0 \leqslant a \leqslant b \leqslant 1\}$ on $X$. Let $\mu$ be the restriction of the Lebesgue measure to the sigma-field $\mathscr{G}$ generated by $\mathscr{F}$. Then $\mu$ is nonatomic on $\mathscr{G}$, but the set $[0,3 / 8] \cup[5 / 8,1] \in \mathscr{G}$ is clearly a $\mu$-atom.

Let $Y=\{0,1\}^{\mathrm{N}_{0}}$, the Cantor space of all sequences of 0's and 1's, equipped with the product topology, where each coordinate space $\{0,1\}$ is equipped with the discrete topology. $Y$ is a perfect, compact, totally disconnected space (see Sikorski (1964) (A) and (B) on p. 28). Let $\mathscr{C}$ be the field of all clopen subsets of $Y$. Then $\mathscr{C}$ is a countable atomless Boolean algebra and any atomless countable Boolean 
algebra is isomorphic to $\mathscr{C}$ (see Sikorski (1964) $(C)$ on p. 28). For every finite sequence of 0 's and 1 's, say, $i_{1}, i_{2}, \ldots, i_{k}$, define

$$
C_{i_{1}, i_{2} \ldots, i_{k}}=\left\{i_{1}\right\} \times\left\{i_{2}\right\} \times \ldots \times\left\{i_{k}\right\} \times\{0,1\} \times\{0,1\} \times \ldots .
$$

Then $\left\{C_{i_{1}, i_{2}, \ldots, i_{k}}: k \geqslant 1\right.$ and $i_{1}, i_{2}, \ldots, i_{k}$ is any finite sequence of 0 's and 1 's $\}$ is a tree in $\mathscr{C}$.

THEOREM 1. Let $B$ be a Boolean algebra. The following statements are equivalent.

(i) There is a nonatomic charge on $B$.

(ii) $B$ contains a tree.

(iii) $B$ has a countable atomless subalgebra $A$.

(iv) The Stone space $X$ of $B$ contains a perfect subset.

(v) $B$ contains an ideal I such that $B / I$ is an atomless Boolean algebra.

Proof. (i) implies (ii) is obvious.

(ii) implies (iii). Let $\left\{b_{i_{1}, i_{2}, \ldots, i_{k}}: k \geqslant 1\right.$ and $i_{1}, i_{2}, \ldots, i_{k}$ is any finite sequence of 0 's and 1 's $\}$ be a tree in $B$. Let $A$ be the Boolean algebra generated by this tree. Note that $A$ is comprised of finite disjoint joins of elements of the tree. Then $A$ is a countable atomless subalgebra of $B$.

(iii) implies (iv). Let $X$ be the Stone space of $B$. In view of the observation made in the paragraph preceding Theorem 1 , we may identify $A$ with $\mathscr{C}$. Consequently, there is a natural homomorphism from the Boolean algebra $\mathscr{C}$ into $B$ which is also one-to-one. Hence there exists a continuous function $f$ from the Stone space $X$ of $B$ onto the Stone space $Y$ of $\mathscr{C}$ (for this result see the discussion in Sikorski (1964), first three paragraphs on $p .34$ ).

Now, we claim that there exists a minimal closed set $P$ contained in $X$ such that $f(P)=Y$. The collection $\mathscr{E}=\{E \subset X: E$ is closed in $X$ and $f(E)=Y\}$ is nonempty and is partially ordered by set inclusion. Let $\left\{E_{\alpha}: \alpha \in D\right\}$ be a chain in $\mathscr{E}$. Then we claim that $\cap_{\alpha \in D} E_{\alpha} \in \mathscr{E}$. For, let $y \in Y$. There exists $x_{\alpha} \in E_{\alpha}$ such that $f\left(x_{\alpha}\right)=y$. Since $X$ is compact, there exists a subnet of $\left\{x_{\alpha}: \alpha \in D\right\}$ converging to some element $x$ in $X$. Since $\left\{E_{\alpha}: \alpha \in D\right\}$ is a chain in $\mathscr{E}$, we have $x_{\beta} \in E_{\alpha}$ if $\beta \geqslant \alpha$. It is therefore clear that $x \in E_{\alpha}$ for every $\alpha \in D$ and $f(x)=y$. By Zorn's lemma there exists a minimal closed set $P \subset X$ with the property that $f(P)=Y$.

We also claim that $P$ is a perfect set. Suppose not. Let $x$ be an isolated point of $P$. Since $P-\{x\}$ is compact, we have that $f(P-\{x\})=Y-\{f(x)\}$ is compact. Hence $f(x)$ is an isolated point of $Y$. This implies that $Y$ is not perfect, and we have a contradiction.

(iv) implies (i). If $X$ contains a perfect subset, by Theorem 1 of Knowles (1967), p. 64 , there exists a regular nonatomic measure on the Borel sigma-field of $X$. By the lemma in Berberian (1962, p. 221), the restriction of this nonatomic measure to the Baire sigma-field of $X$ is also nonatomic. By the Stone-Weierstrass theorem the field $\mathscr{F}$ of all clopen subsets of $X$ generates the Baire sigma-field on $X$ (see 
Halmos (1950), Exercise (7), p. 223). By the lemma at the beginning of this section, the restriction of this nonatomic measure on the Baire sigma-field on $X$ to $\mathscr{F}$ is nonatomic. Since $\mathscr{F}$ and $B$ are isomorphic, we may transfer this restriction to $B$, and thereby obtain a nonatomic charge on $B$.

(i) implies (v). Let $\mu$ be a nonatomic charge on $B$. Let $I$ be the ideal of all $\mu$-null elements of $B$. Then $B / I$ is an atomless Boolean algebra.

(v) implies (iv). Let $Z$ be the Stone space of $B / I$. Since $B / I$ is atomless, $Z$ is perfect (see Sikorski (1964) $(A)$ on p. 28). Since the natural homomorphism from $B$ to $B / I$ is onto, there exists a continuous function $f$ from $Z$ to $X$ which is one-to-one (see Sikorski (1964), first three paragraphs on p. 34). Then $f(Z)$ is a perfect subset of $X$.

COROLlary 1. There exists a nonatomic charge on every infinite Boolean sigmaalgebra.

Proof. Let $B$ be an infinite Boolean sigma-algebra. Let $b_{1}, b_{2}, \ldots$ be a sequence of nonzero pairwise disjoint elements in $B$ such that $\bigvee_{i \geqslant 1} b_{i}=1$. Let $N$ be the set of positive integers. Let $N_{0}$ and $N_{1}$ be a decomposition of $N$ into two infinite disjoint subsets. Define $b_{0}=\bigvee_{i \in N_{0}} b_{i}$ and $b_{1}=\bigvee_{i \in N_{1}} b_{i}$. Applying this technique of decomposition at every stage we can easily obtain a tree in $B$. Theorem 1 completes the proof.

A Boolean algebra $B$ is said to be superatomic if the Stone space $X$ of $B$ is scattered, that is, if no subset of $X$ is perfect (see Sikorski (1964), p. 35).

Corollary 2. A Boolean algebra $B$ is superatomic if and only if there is no nonatomic charge on $B$.

Proof. Use the equivalence of (i) and (iv) of Theorem 1.

REMARK. The collection of all countable Boolean algebras which admit no nonatomic charges (that is, which are superatomic) has been characterized by Mazurkiewicz and Sierpinski. Each such algebra is isomorphic to the field of all clopen subsets of $[0, \alpha]$, for some countable ordinal $\alpha$ (see the introduction in Pierce (1970), p. 1).

\section{Conditions for the denseness of the set of all nonatomic probability charges in the space of all probability charges}

Let $B$ be a Boolean algebra and let $\mathscr{P}$ be the collection of all probability charges on $B$. Equip $\mathscr{P}$ with a topology by defining the convergence as follows. A net $\mu_{\alpha}$ of probability charges in $\mathscr{P}$ converges to a probability charge $\mu$ if $\mu_{\alpha}(b)$ converges to $\mu(b)$ for every $b$ in $B$. Let $X$ be the Stone space of $B$, and let $\mathscr{R}$ be the collection of 
all regular probability measures on the Borel sigma-field on $X$. We equip $\mathscr{R}$ with the weak $*$ topology, that is, a net $\eta_{\alpha}$ in $\mathscr{R}$ converges to an $\eta$ in $\mathscr{R}$ if $\int f d \eta_{\alpha}$ converges to $\int f d \eta$ for every real-valued continuous function $f$ on $X$ (see Knowles (1967), Section 2). Corresponding to every charge $\mu$ in $\mathscr{P}$ we associate a (unique) measure $\eta$ in $\mathscr{R}$ as follows. Using the isomorphism between $B$ and the field $\mathscr{F}$ of all clopen sets of $X$ we may transfer $\mu$ from $B$ to $\mathscr{F}$. Let $\mu_{1}$ denote this charge on $\mathscr{F}$. Since any charge on $\mathscr{F}$ is a measure, we may extend $\mu_{1}$ to a measure $\eta_{1}$ on the Baire sigma-field of $X$. Then $\eta_{1}$ can be extended uniquely to a regular Borel measure $\eta$ on the Borel sigma-field on $X$. In fact, this correspondence $T$ between $\mathscr{P}$ and $\mathscr{R}$ is a homeomorphism. This follows from the fact that, by the Stone-Weierstrass theorem, the collection of all finite linear combinations of indicator functions of clopen subsets of $X$ is dense in the space of all continuous functions on $X$ equipped with the supremum norm.

THEOREM 2. Let $B$ be a Boolean algebra. The following statements are equivalent.

(i) The collection of all nonatomic probability charges on $B$ is dense in $\mathscr{P}$.

(ii) $B$ is atomless.

(iii) The Stone space of $B$ is perfect.

Proor. The equivalence of (ii) and (iii) is well known (see Sikorski (1964), (A) on p. 28).

(i) implies (ii). Suppose that $B$ contains an atom $b$. Let $\mu$ be the unique probability charge supported on $b$, that is, $\mu(d)=1$ if $b \leqslant d$ and $\mu(d)=0$ otherwise. Since (i) holds, there exists a net $\mu_{\alpha}$ of nonatomic probability charges converging to $\mu$. But because $b$ is an atom we must have $\mu_{\alpha}(b)=0$ for every $\alpha$, while $\mu(b)=1$. This contradiction shows that there can be no atoms in $B$.

(iii) implies (i). Knowles $(1967$, p. 65) remarked that if $X$ is perfect, the set $Q$ of all nonatomic regular probability measures on the Borel sigma-field of $X$ is a dense $G_{\delta}$ subset of $\mathscr{R}$. By the lemma proved in Section $3, T^{-1} Q \subset$ the set of all nonatomic probability charges on $B$. So (i) follows.

Using Theorem 2 we prove the following result.

TheOREM 3. Let $B$ be a Boolean algebra and let $\mathscr{P}$ be the set of all probability charges on $B$. Let $I=\cap_{\mu} I_{\mu}$, where the intersection is taken over the set $\mathscr{I}$ of all nonatomic charges $\mu$ in $\mathscr{P}$ and the set $I_{\mu}$ is the ideal of all $\mu$-null elements in $B$. Then the closure of $\mathscr{I}$ in $\mathscr{P}$ is the set of all charges in $\mathscr{P}$ which vanish on $I$.

Proof. First, we note that $B / I$ is atomless. For, if the equivalence class containing $b$, in $B / I$, is not equal to $I$, then $b \notin I$. There exists a $\mu$ in $\mathscr{I}$ such that $\mu(b)>0$. We can find $d \in B$ such that $d \leqslant b$ and $0<\mu(d)<\mu(b)$. Clearly $I \neq[d]<[b]$. Now 
note that the collection of all probability charges on $B$ which vanish on $I$ is homeomorphic to the collection of all probability charges on $B / I$. Under this homeomorphism a nonatomic probability charge on $B$ corresponds to a nonatomic probability charge on $B / I$. Theorem 2 completes the proof.

\section{Acknowledgements}

The authors are grateful to the referee for his invaluable advice in substantially improving the paper. Theorem 3 and condition (v) in Theorem 1 were suggested by him.

\section{References}

S. K. Berberian (1962), Measure and Integration (Macmillan, New York).

P. R. Halmos (1950), Measure Theory (Van Nostrand, New York).

P. R. Halmos (1963), Lectures on Boolean Algebras (Van Nostrand, New York).

J. D. Knowles (1967), On the existence of nonatomic measures, Mathematika 14, 62-67.

K. Kuratowski (1966), Topology 1 (Academic Press, New York).

N. Y. Luther (1970), "Weak denseness of nonatomic measures on perfect, locally compact spaces", Pacific J. of Math. 34, 453-460.

R. S. Pierce (1970), "Existence and uniqueness theorems for extensions of zero-dimensional compact metric spaces", Trans. Amer. Math. Soc. 148, 1-21.

R. Sikorski (1964), Boolean Algebras (Springer-Verlag, New York).

A. E. Taylor (1958), Introduction to Functional Analysis (Wiley, New York).

Mathematical Statistics

Indian Statistical Institute

Calcutta

India

Department of Probability and Statistics

The University of Sheffield

Sheffield

U.K. 